\title{
Heterogeneity of late-infantile neuronal ceroid lipofuscinosis
}

Nanbert Zhong, $M D^{1,2,4}$, Dorota N. Moroziewicz, $M S^{1,2}$, Weina Ju, $M S^{1,2}$, Anna Jurkiewicz, $P h D^{1,2}$, Lance Johnston, $B S^{5}$, Krystyna E. Wisniewski, $M D, P h D^{3,4}$, and W. Ted Brown, $M D, P h D^{2}$

\begin{abstract}
Purpose: Late-infantile neuronal ceroid lipofuscinosis (LINCL), an autosomal recessively inherited lysosomal storage disorder characterized by autofluorescent inclusions and rapid progression of neurodegeneration, is due to CLN2 gene mutations. However, CLN2 mutation analysis has failed to identify some clinically diagnosed "late-infantile" NCL cases. This study was conducted to further characterize genetic heterogeneity in families affected by LINCL. Methods: DNA mutations in the CLN1, CLN2, and CLN3 genes that underlie INCL (infantile NCL), LINCL, and JNCL (juvenile NCL), respectively, were studied with molecular analyses. Results: A total of 252 families affected by childhood NCL were studied. Of 109 families clinically diagnosed as having LINCL, 3 were determined to have either INCL or JNCL by identification of mutation(s) in CLN1 or CLN3. Six families diagnosed initially as having JNCL were found to have LINCL based on the finding of mutations in the CLN2 gene. In addition, several novel mutations were identified. Conclusions: Clinical and genetic heterogeneity of LINCL was demonstrated in nine LINCL families studied. Genetics in Medicine, 2000:2(6):312-318.
\end{abstract}

Key Words: neuronal ceroid lipofuscinosis, late-infantile neuronal ceroid lipofuscinosis, CLN genes, mutation analyses, genetic heterogeneity

The neuronal ceroid lipofuscinoses (NCLs), commonly referred to as Batten disease, are a group of neurodegenerative disorders in humans and animals. The NCLs comprise eight forms classified initially by clinical symptoms and age at onset but now recognized as being due to mutations in genes $C L N 1-8 .{ }^{1}$ In addition to an adult-onset form (ANCL, or Kuf's disease, OMIM: 204300), the childhood-onset forms include three classical forms, i.e., infantile (INCL, OMIM: 256730), late-infantile (LINCL, OMIM: 204500), and juvenile (JNCL, OMIM: 204200); and four variants, the "Finnish" variant LINCL (fLINCL, OMIM: 256731), "Portugal" LINCL (pLINCL, OMIM: 601780), "Turkish" LINCL (tLINCL, OMIM: pending), and progressive epilepsy with mental retardation (EPMR, also called northern epilepsy, OMIM: 600143). The incidence of the NCLs is estimated at approximately 1 to 5 in 100,000 birth in the general population' ${ }^{2}$ and as high as 1 in 12,500 to 1 in 20,000 as a result of founder effects in genetically isolated populations such as the Finnish, ${ }^{3}$ which gives a figure of 1 to 8 per 100,000 globally. In the United States, 440,000 families have been reported as affected by the NCLs, ${ }^{4}$ a figure which appears to be an overestimate. Currently, we estimate that ap-

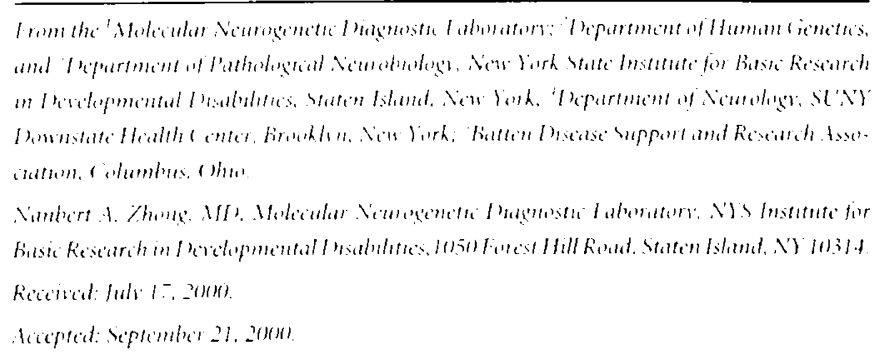

proximately 25,000 families in the United States are affected by NCLs. Regardless of the true prevalence, the NCLs are regarded as the most common group of pediatric neurodegenerative disorders.

Clinically, NCLs are characterized by rapid deterioration of vision, seizures, progressive development of mental retardation, movement disorders, and behavioral changes. ${ }^{5}$ Brain atrophy, as documented by magnetic resonance imaging (MRI) and computed tomography (CT), is most pronounced in the supratentorium and brainstem. ${ }^{6}$ Cerebellar atrophy which results in enlargement of the $4^{\text {th }}$ ventricle, without concomitant cerebral atrophy, may be an early sign of NCL, and such abnormalities are detectable even before clinical symptoms are apparent. $^{i-9}$

Pathologic studies of all forms of NCLs have revealed unique electron microscopic profiles of lysosomal lipopigmental storage materials, the lipofuscins, ${ }^{10}$ which include fingerprint (FP), granular osmiophilic deposits (GRODs), curvilinear (CV), and rectilinear $(\mathrm{RL})$ inclusions. In typical NCL cases, GRODs are present in INCL, CV in LINCL, and FP in JNCL. However, the profiles can vary, and "mixed" profiles can occur in atypical cases. ${ }^{11.12}$

Biochemical abnormalities involving lysosomal hydrolases have been shown in the NCLs. ${ }^{13}$ Mitochondrial ATPase subunit $C$ is the predominant storage material in all NCLs except INCL. In INCL, the accumulated material is composed of saposins (SAPs) A and D, which also are observed to accumulate in fLINCL and PEMR (Table 1). ${ }^{14-16}$ The NCL group of neurodegenerative disorders is now considered to be a new class of lysosomal storage diseases caused by genetic deficiency of ly- 
Table 1

Classification of the neuroceroid lipofucinoses

\begin{tabular}{lcll}
\hline NCL & $\begin{array}{c}\text { Gene } \\
\text { designated }\end{array}$ & Ultrastructural finding by EM & \multicolumn{1}{c}{ Material stored } \\
\hline Infantile (INCL) & $C L N 1$ & GROD & SAPs (A and D) \\
Late-infantile (LINCL) & $C L N 2$ & CV, Mixed & Subunit C of ATPase \\
Iuvenile (JNCL) & $C L N 3$ & FP, Mixed & Subunit C of ATPase \\
Adult (ANCL) & $C L N 4$ & Mixed & Subunit C of ATPase \\
Finnish (fLINCL) & $C L N 5$ & FP, CV, RL & Subunit C of ATPase SAPs (A and D) \\
Portugal (pLINCL) & $C L N 6$ & CV, FP, RL & Subunit C of ATPase \\
Turkish (tLINCL) & $C L N 7$ & FP, Mixed & Subunit C of ATPase \\
Progressive epilepsy with mental retardation (EPMR) & $C L N 8$ & CV- or GROD-like inclusion & Subunit C of ATPase SAPs (A and D) \\
\hline
\end{tabular}

EM, electron microscopy; GROD, osmiophilic deposits; CV, curvilinear; FP, fingerprint; RL, rectilinear; SAPs, saposins; ATPase, mitochondrial ATP synthase; NCL, neuroceroid lipofucinosis.

sosomal proteases as well as dysfunction of structural proteins. ${ }^{1,17}$ No treatment is available at present, and the outcome of these diseases is usually fatal a few years after the onset of clinical symptoms.

Classical LINCL is characterized by the onset of seizures and progressive dementia between 2 and 4 years of age. Ataxia and myoclonus are constant features. Visual failure usually appears after the onset of neurological symptoms. Macular and retinal degeneration plus optic atrophy causes blindness by 5 or 6 years. Death usually occurs between 6 and 15 years. The characteristic ultrastructural finding in LINCL is "pure CV profiles," which do not contain the GRODs or FP components seen in INCL and JNCL. The mature form of subunit $\mathrm{C}$ of the mitochondrial ATP synthase, is the main component of the storage lipofuscins. ${ }^{15}$ The highly hydrophobic nature of this proteolipid explains the insolubility of the storage material.

LINCL is encoded by the gene CLN2, which maps ${ }^{18}$ to chromosome $1 \mathrm{lpl} 15$ and consists of 13 exons spanning $6.65 \mathrm{~kb}$ of genomic DNA. ${ }^{19}$ This gene encodes a $46-\mathrm{kD}$ protein, the lysosomal pepstatin-insensitive lysosomal peptidase (LPIP) or tripeptidyl peptidase 1 (TPP1), which cleaves tripeptides from amino termini of peptides that bear free $\alpha$-amino groups. ${ }^{20}$ Mutations in CLN2 abolish TPP1 enzymatic activity in LINCL patients. A total of 31 mutations have been identified. The mutations are distributed relatively evenly across the 13 exons, although there are no mutations in the first two exons. We reported that certain missense mutations in CLN2 resulted in atypical juvenile onset. ${ }^{21}$ Two of these missense mutations, 636 $\mathrm{C} \rightarrow \mathrm{T}(\mathrm{R} 280 \mathrm{X})$ and T523-1 G $\rightarrow \mathrm{C}$ (IVS 5-1), were found to be common. ${ }^{22}$ DNA-based genetic testing for these mutations detects approximately $70 \%$ of LINCL families. ${ }^{23}$ Some, but not all of the remaining $30 \%$ consist of cases of classical LINCL resulting from TPP1 deficiency caused by uncommon mutations. Our previous studies showed that the $\mathrm{R} 447 \mathrm{H}$ mutation in $C L N 2$, along with one of the common mutations described above, causes atypical juvenile onset of NCL. ${ }^{24}$ In this study, we analyzed $252 \mathrm{NCL}$ families, including 34 clinically diagnosed affected with INCL, 109 with LINCL, and 98 with JNCL for clinical and genetic heterogeneity.

\section{MATERIALS AND METHODS}

\section{NCL families}

Clinically diagnosed NCL families were either obtained from the George Jervis Clinic at our Institute or referred by the Batten Disease Support and Research Association (BDSRA) or by clinicians nationally and internationally. Clinical information, including genetic ethnic background, age at onset, neurological assessment for vision loss/blindness, learning and speaking disabilities, walking difficulties/inability to walk, behavioral problems and dementia, and seizures/convulsions, as well as ultrastructural studies with electronic microscopic examinations, was collected. Genetic studies were performed only for participants from whom informed consent was obtained, and clinical diagnostic results were shared with participants.

\section{DNA samples}

Genomic DNA was extracted by using a Puregene kit (Gentra, NC), either directly from peripheral whole blood or from banked lymphocytes and/or fibroblasts, derived from affected NCL probands and family members or normal controls.

\section{Molecular analyses}

Polymerase chain reaction (PCR) -based molecular analyses for five common mutations in genes $C L N 1, C L N 2$, and CLN3 were conducted with protocols previously published. ${ }^{22-24}$ "Gene-scan" procedures to search for uncommon mutations in the entire CLN2 gene were performed as described elsewhere. ${ }^{25}$ Briefly, four genomic fragments that include the entire CLN2 coding sequence, all exon-intron junctions, some intron sequences, and a 500-bp promoter region ${ }^{19}$ were PCR-amplified. Fragment 1 (1.3-kb) contains the 500-bp promoter region and exons 1-3; fragment $2(0.9-\mathrm{kb})$ contains exons $4-6$; fragment $3(0.9-\mathrm{kb})$ contains exons 7-9; and fragment 4 (1.2-kb) contains exons 10-13. The PCR product was gel purified and sequenced from both directions. ${ }^{26}$ 


\section{RESULTS}

\section{LINCL is the second most common NCL globally}

Among 1,960 families worldwide affected by NCLs and registered with BDSRA, LINCL accounts for $25.9 \%$, second to JNCL with $46.4 \%$ (Table 2). The frequency of classical childhood onset INCL, LINCL, and JNCL, together account for $89 \%(1,745$ of 1,960$)$ of the NCL cases. In the United States, the frequency of LINCL is close to the global distribution due to the American population being more heterogeneous than ge- netically homogeneous populations such as the Finnish, in which INCL is the second-most common NCL and accounts for almost $40 \%$ (159 of 398 ) of cases.

\section{Molecular analyses of $\mathbf{2 5 2}$ clinically referred cases of childhood NCL}

Two hundred fifty-two families, diagnosed clinically as affected by childhood-onset NCLs, were referred to our laboratory in the past 2 years. One hundred thirty-six cases were from 47 institutions nationwide, and 86 cases were from 11 institu-

Table 2

Global distribution of neuroceroid lipofucinoses

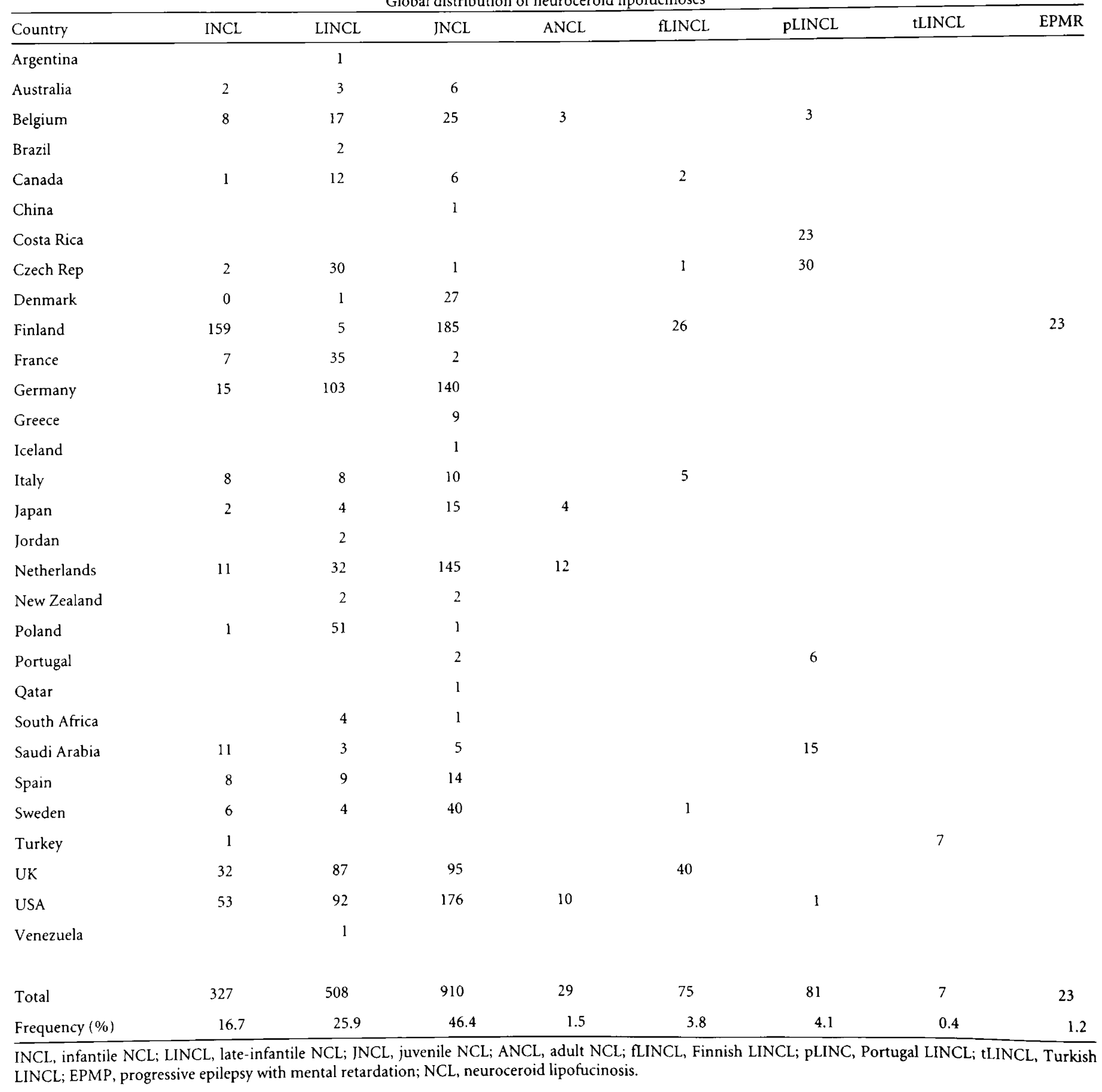


tions in eight countries internationally. Among these cases, $43.3 \%$ ( 109 of 252 ) were diagnosed as having LINCL. Molecular analyses of the clinically referred LINCL cases, revealed that testing for the two common mutations detected only about $60 \%$ (65 of 109) of LINCL cases, lower than the expected figure of $70 \%$ we described earlier in LINCL cases diagnosed by electron microscopy. Among 30\% (33 of 109) of cases in which one allele has been found to carry one of the common mutations, seven novel mutations, including a nonsense mutation (W460X), four missense mutations (R127Q, G284 V, G473R, and A555P), an intron-exon junction mutation (IVS4-2A \&U279D; G), and a single base "T" insertion that results in L120S in exon 4 and frame shifted thereafter (Table 3) were identified in addition to uncommon mutations described elsewhere. ${ }^{27}$ Thirty-three percent (36 of 109) of clinically diagnosed cases of LINCL could not be identified as mutations involving the CLN1-3 genes (Table 4). This result indicates that either this group of LINCL cases is not NCL, or they are the result of mutations in the CLN5-8 genes encoding variant forms of LINCL, or they result from other unidentified genetic factors.

\section{Documentation of clinic and genetic heterogeneity in LINCL}

Three cases (LINCL1-3), clinically diagnosed as having "late-infantile" NCL on the basis of age at onset, were affected by either INCL or JNCL, with mutations found in CLN1 or CLN3 genes. Six families (JNCL1-6) that had been diagnosed initially with "juvenile" NCL were found to have LINCL with mutations in the CLN2 gene (Table 5). Clinically, all the JNCLsl-6 presented with a juvenile onset by the age of 5 to 6 years and showed no indications for classical LINCL, either by the initial symptoms or by ultrastructural profiles. The proband JNCLl presented with slow learning as the initial symptom having EM findings with predominantly a $\mathrm{CV}$ profile, along with some FP and GRODs. There were no vision problems at any time, but seizures and motor dysfunction have been progressive over 5 years. Three cases (JNCL2-4) were initially diagnosed as JNCL on the basis of vision problems. However, EM results showed a mixed profile of FP, CV, and/or GRODs, findings which usually result in diagnostic difficulties.
Progression in these cases was slow and life spans for JNCL3-4 are longer than typical for both JNCL3 and JNCL4, which may survive for more than 15 to 20 years after the initial onset of clinical symptoms. Molecular genetic analyses of the CLN3 gene showed no mutation(s) that could be detected. However, they were determined to carry either one or both common mutation(s) of the CLN2 gene. In addition, a mutation in the CLN1 gene was found in a "juvenile" NCL family (JNCL7, not shown in Table 5). These data indicated the presence of genetic heterogeneity in that similar symptoms, such as the clinical onset of late-infantile, may result from mutation(s) in either genes $C L N 1$ or $C L N 3$ in addition to CLN2. This demonstrated the presence of clinical heterogeneity in LINCL, since various mutation(s) in CLN2 may lead to different clinical features, such as onset in infantile or juvenile age ranges, in addition to the classical late-infantile age.

\section{DISCUSSION}

Our studies provide evidence for genetic heterogeneity underlying LINCL. Three cases diagnosed initially on the basis of clinical features, including the age-at-onset being of the lateinfantile range (cases LINCL-2 and LINCL-3), or the initial symptoms including seizures (LINCL-3), or the CV pathologic profile (LINCL-1), showed no detectable mutations in the $C L N 2$ gene. However, mutations were found in genes CLN1 and CLN3. Likewise, six cases with juvenile onset and ultrastructural findings of the FP profile were shown to have mutations in gene CLN2. Although these nine cases account for $3.6 \%$ of 252 families studied with childhood onset, they account for $2.8 \%$ (3 of 109) of clinically diagnosed LINCL and $6.1 \%$ ( 6 of 98 ) clinically diagnosed JNCL cases, which indicates that genetic heterogeneity is present in LINCL. These data suggest that genetic heterogeneity for LINCL may present either in that LINCL may result from mutations in CLN genes other than CLN2 or mutations in the CLN2 gene may result in clinically variant features misinterpretable as other NCL forms. This is important when molecular testing is offered for LINCL. If no mutation is found in clinically referred LINCL cases, a search for mutations in CLN1 and CLN3 genes and biochem-

Table 3

Novel mutations identified at the second allele in CLN2 gene

\begin{tabular}{lllll}
\hline $\begin{array}{l}\text { Age at } \\
\text { onset }(\mathrm{yr})\end{array}$ & \multicolumn{1}{c}{ Initial symptom } & EM & $\begin{array}{c}\text { Mutation at } \\
\text { allele-1 }\end{array}$ & $\begin{array}{c}\text { Mutation at } \\
\text { allele-2 }\end{array}$ \\
\hline $2-2.5$ & Seizures & $\mathrm{CV}$ & R280X & G473R \\
3 & Seizures & $\mathrm{CV}$ & R280X & IVS4-2G \\
4 & Seizures & $\mathrm{CV}$ & IVS5-1C & W460X \\
3 & Speech delay, seizures & $\mathrm{CV}$ & IVS5-1C & A555P \\
$2-2.5$ & Speech delay, seizures & NA & IVS5-1C & I-bp insertion \\
2 & Behavioral changes & CV & IVS5-1C & R127Q \\
4 & Vision problem & Mix & IVS5-1C & \\
\hline
\end{tabular}

EM, electron microscopy findings; CV, curvilinear profile; FS, frame shift; NA, data are not available; Mix, mixed profile containing CV/FP/GRODs. 
Table 4

Families affected by childhood neuroceroid lipofucinoses

\begin{tabular}{|c|c|c|c|c|}
\hline \multirow[b]{2}{*}{ Mutation } & \multicolumn{4}{|c|}{ NCL family ( $N=252$, distribution $\%)$} \\
\hline & $\begin{array}{c}\text { INCL } \\
(n=34)\end{array}$ & $\begin{array}{c}\text { LINCL } \\
(n=109)\end{array}$ & $\begin{array}{c}\text { JNCL } \\
(n=98)\end{array}$ & $\begin{array}{c}\text { Unclassified NCL } \\
\quad(n=11)\end{array}$ \\
\hline $\mathrm{Cm} / \mathrm{Cm}$ & $9(26.5)$ & $32(29.4)$ & $47(48.0)$ & \\
\hline $\mathrm{Cm} / \mathrm{Um}$ & $10(29.4)$ & $33(30.3)$ & $18(18.4)$ & \\
\hline $\mathrm{Um} / \mathrm{Um}$ & $5(14.7)$ & $5(4.6)$ & 0 & \\
\hline M@L & 0 & $3(2.8)$ & $7(7.1)$ & \\
\hline Unidentified & $10(29.4)$ & $36(33.0)$ & $26(26.5)$ & $11(100)$ \\
\hline
\end{tabular}

$\mathrm{Cm}$, common mutation(s); Um, uncommon mutation(s); M@L, mutations were found at another NCL locus; Unidentified, no mutations could be detected in the underlying gene; Unclassified, cases could not be classified on the basis of clinical features; NCL, neuroceroid lipofucinosis.

ical analysis for enzymatic activity for the CLN2-encoded TPP $1^{28}$ is recommended.

Although classical LINCL presents as the second most common form of NCLs, four other variants with late-infantile onset, fLINCL, pLINCL, tLINCL, and EPMR, have been identified and characterized. ${ }^{29-32}$ In addition, mutations may occur in the CLN1 or CLN3 genes, and the CLN5-8 genes underlying these four variants should not be ignored. The fLINCL and PEMR so far have only been found in the Finnish population (Table 2). Although there were no mutations identified in the CLN2 gene in 36 American cases (33\%) (Table 4), which are predominantly of European descent, it is possible that genetic deficiency in the CLN5 or CLN8 may occur in these or in other populations globally.

Seizures, as the initial symptom, may present in the other two LINCL variants, the PLINCL and tLINCL that are encoded by gene CLN6 and CLN7, respectively. The pLINCL is characterized clinically by a slightly later onset than classical LINCL and pathologically by mixed profile $(\mathrm{CV}+\mathrm{FP})$ inclusions. The age of onset ranges from 18 months to 8 years. The leading symptoms are seizures, motor delay, dysarthria, and ataxia. In $50 \%$ of cases, visual failure occurs early on. Most patients die between the ages of 5 and 12 years. ${ }^{33}$ The CLN6-encoded LINCL variant has been assigned to $\mathrm{PLINCL}^{1}{ }^{1}$ because the majority of cases of pLINCL seem to be from Portugal, India or Pakistan, and of Czech-Romany descent. The Romany population is thought to originate from India/Pakistan, indicating there is a close ethnic link for this LINCL variant. The 23 cases of pLINCL from the Costa Rican population listed in Table 2 were likely to have originated from Portugal or India/Pakistan (doi:10.1006/mgme.2000.3057, http://www.idealibrary. com). ${ }^{34}$ The gene CLN7 for Turkish LINCL (tLINCL) has not been localized and identified. The limited data for tLINCL, which were collected from small numbers of cases, showed tLINCL is Turkish originated with onset of symptoms in the late-infantile range. At the early stages of the disease, seizures and poor mobility are prominent features. Visual impairment has a variable onset. Subsequent symptoms include motor and cognitive deterioration. Pathologically, FP and CV may be present. To characterize the $33 \%$ of unidentified LINCLs, further studies on genes CLN5-8 need to be conducted.

Genotype and phenotype for the novel mutations (Table 4) showed correlation. Three cases with seizures as the initial symptom suggest that the seizures might be related to muta-

Table 5

Heterogeneity of late-infantile neuroceroid lipofucin

\begin{tabular}{|c|c|c|c|c|c|}
\hline $\begin{array}{l}\text { Clinical } \\
\text { diagnosis }\end{array}$ & $\begin{array}{c}\text { Age at } \\
\text { onset }(\mathrm{yr})\end{array}$ & Initial symptom & EM & $\begin{array}{c}\text { Gene } \\
\text { mutated }\end{array}$ & $\begin{array}{c}\text { Genetic } \\
\text { diagnosis }\end{array}$ \\
\hline LINCL-1 & 6 & Learning delay & $\mathrm{CV} / \mathrm{FP}$ & CLN3 & JNCL \\
\hline LINCL-2 & 1.4 & Vision loss & NT & CLN1 & INCL \\
\hline LINCL-3 & 3.5 & Seizures & FP & CLNI & INCL \\
\hline JNCL-1 & 6 & Learning delay/seizures & CV/FP/GRODs & CLN2 & LINCL \\
\hline JNCL-2 & 5 & Vision problem & FP/CV/GRODs & CLN2 & LINCL \\
\hline JNCL-3 & 6 & Vision \& dementia & FP/GRODs & CLN2 & LINCL \\
\hline JNCL-4 & 6 & Vision problem & $\mathrm{FP} / \mathrm{CV}$ & CLN2 & LINCL \\
\hline JNCL-5 & 6 & Behavior & $\mathrm{FP} / \mathrm{CV}$ & CLN2 & LINCL \\
\hline JNCL-6 & 5 & Walking problem & FP & CLN2 & LINCL \\
\hline
\end{tabular}

NT, EM study was not performed; LINCL, late-infantile neuroceroid lipofucin; JNCL, juvenile NCL; EM, electron microscopy; CV, curvilinear; FP, fingerprint; GRODs, osmiophilic deposits. 
tions that involve in exon 11. These mutations resulted in either a substitution of amino acid residue (G473R) that also associated with ocular motor apraxia, ${ }^{35}$ premature termination of the peptide (W460X), or intron-exon junction errors leading to an alternative splicing, which resulted in lack of exon 11. Vision problems in LINCL are not a typical clinical feature and usually have a late stage of onset. In the case with vision problems studied here, the phenotype may be related to the pathologic changes of FP/GRODs, which are always found in classical JNCL that has pure FP or atypical JNCL that has mixed FP with GRODs. This may apply to any LINCL case as in JNCL-2 and JNCL-5 listed in Table 5, in which FP/GRODs are found. Because of the overlapping clinical symptoms, clinically diagnosed JNCL may instead be due to mutations in gene CLN2 (LINCL). Thus, clinical heterogeneity of LINCL should be considered.

Currently, the amount of molecular heterogeneity for LINCL remains unknown. The correlation between the mutations in a specific genetic locus and the pathologic process is not clear yet. There is no answer to the question why different mutations in different loci may result in a similar pathologic change of lipofuscin storage. We have hypothesized ${ }^{36}$ that a common pathogenic pathway may present, in which there is an unidentified mechanism. Characterization of this unknown mechanism may help in better understanding the NCLs and may guide in designing a rational treatment for the NCLs. As described earlier, INCL and LINCL result from lysosomal enzyme deficiencies that may be corrected by the supplement of normal PPT1 or TPP1. Definite diagnosis of INCL and LINCL by genetic testing for mutation(s) in the $C L N 1$ or $C L N 2$ gene can provide basic information for the families, not only for presymptomatic or prenatal diagnosis, but also for consideration of possible enzyme replacement therapy. However, in JNCL, because Battenin is a membranous protein, treatment by such means may be more difficult. However, a deeper understanding of Battenin's function in the pathogenic process may provide the information required for designing a treatment.

\section{Acknowledgments}

This study was supported in part by grants from the New York State Office of Mental Retardation and Developmental Disabilities, the Batten Disease Support and Research Foundation, and the Children's Brain Disease Foundation.

\section{References}

1. Zhong N. Neuronal ceroid lipofuscinoses and possible pathogenic mechanism. Mol Genet Metabol 2000;71:195-206.

2. Zeman W. Studies in the neuronal ceroid lipofuscinoses. INeuropathol Exp Neurol 1974:33:1-12.

3. Mitchison HM, O'Rawe AM, Taschner PEM, Sandkuijl LA, Santavuori P, de Vos N, Breuning MH, Mole SE, Gardiner RM, Jarvela IE. Batten disease gene, CLN3: linkage disequilibrium mapping in the Finnish population, and analysis of European haplotypes. Am J Hum Genet 1995;56:654-662.

4. Rider JA, Rider DL. Batten disease, a twenty-eight-year struggle: past, present and future. Neuropediatrics 1997;28:4-5.

5. Wisniewski KE, Kida E, Connell F, Zhong N. Neuronal ceroid lipofuscinoses: research update. I Ital Neuro Sci 2000;21:\$49-S56.
6. Raininko R, Santavuori P, Heiskala H, Sainio K, Palo J. CT findings in neuronal ceroid lipofuscinoses. Neuropediatrics 1990;21:95-101.

7. Armstrong D, Quisling RG, Webb A, Koppang N. Computed tomographic and nuclear magnetic resonance correlation of canine ceroid-lipofuscinosis with aging. Neurobiol Aging 1983;4:297-303.

8. Petersen B, Handwerker M, Hupperz H-I. Neuroradiological findings in classical late infantile neuronal ceroid-lipofuscinosis. Pediatr Neurol 1996;15:344-347.

9. Vanhanen SL, Raininko R, Autti T, Santavuri P. MRI evaluation of the brain in infantile neuronal ceroid lipofuscinosis. Parts 1 \& 2. I Child Neurol 1995;10:438 450.

10. Zeman W, Donahue S. Fine structure of the lipid bodies in juvenile amaurotic idiocy. Acta Neuropathol 1963:3:144-149.

11. Goebel HH, Mole SE, Lake BD. Introduction. In: Goebel HH, Mole SE, Lake BD, editors. The neuronal ceroid lipofuscinoses (Batten disease). Amsterdam, the Neth erlands: IOS Press, 1999:1-4.

12. Wisniewski KE, Kida E, Patxot OF, Connell F. Variability in the clinical and pathological finding in the neuronal ceroid lipofuscinoses: review of data and observations. Am J Med Genet 1992;42:525-532.

13. Prasad VV, Pullarkat RK. Brain lysosomal hydrolases in neuronal ceroid-lipofuscinoses. Mol Chem Neuropathol 1996;29:169-179.

14. Fearnley IM, Walker IE, Martinus RD, Jolly RD, Kirkland KB, Shaw GJ, Palmer DN. The sequence of the major protein stored in ovine ceroid-lipofuscinosis is identical with that of the dicyclohexylcarbodiimide reactive proteolipid of mitochondrial ATP synthase. Biochem J 1990:268:751-758.

15. Palmer DN, Fearnley IM, Walker IE, Hall NA, Lake BD, Wolfe LS, Haltia M, Martnus RD, Boyliss SL, Jolly RD. Mitochondrial ATP synthase subunit $C$ storage in the ceroid-lipofuscinoses (Batten disease). Am I Med Genet 1992;42:561-567.

16. Santavuori P, Rapola J, Haltia M, Tyynela I, Peltonen L, Mole SE. CLN5 Finnish variant late infantile NCL. In: Goebel HH, Mole SE, Lake BD, editors. The neurona ceroid lipofuscinoses (Batten disease). Oxford: IOS Press, 1999:91-101.

17. Bennett MJ, Hofmann SL. The neuronal ceroid-lipofuscinoses (Batten disease): a new class of lysosomal storage disease. J Inherit Metab Dis 1999;22:535-544.

18. Sharp JD, Wheeler RB, Lake BD, Savukoski M, Jarvela IE, Peltonen L, Gardiner RM, Williams RE. Loci for classical and a variant late infantile neuronal ceroid lipofuscinosis map to chromosome 1 lp15 and 15q21-23. Hum Mol Genet 1997;6:591-596.

19. Liu C-L, Sleat DE, Donnelly RJ, Lobel P. Structural organization and sequence of CLN2, the defective gene in classical late infantile neuronal ceroid lipofuscinosis. Genomics 1998;50:206-212.

20. Junaid MA, Pullarkat RK. Increased brain lysosomal pepstatin-insensitive proteinase activity in patients with neurodegenerative disorders. Neurosci Lett 1999;264 157-160.

21. Wisniewski KE, Kaczmarski A, Kida E, Connell F, Kaczmarski W, Michalewski MP Moroziewicz DN, Zhong N. Reevaluation of neuronal ceroid lipofuscinoses: atypical juvenile onset may be the result of CLN2 mutations. Mol Genet Metab 1999;66: $248-252$

22. Zhong N, Wisniewski KE, Hartikainen J, Ju W, Moroziewicz DN, McLendon L, Sklower-Brooks SS, Brown WT. Two common mutations in the CLN2 gene underlie late infantile neuronal ceroid lipofuscinosis. Clin Genet 1998;54:234-238.

23. Zhong N, Wisniewski KE, Kaczmarski AL, Ju W, Xu WM, Xu WW, McLendon L, Liu B, Kaczmarski W, Sklower-Brooks S, Brown WT. Identification of a novel missense mutation (E295K) underlying Batten disease. Hum Genet 1998;102:57-62.

24. Zhong N, Ju W, Moroziewicz DN, Kaczmarski AL, Hartikainen I, Suarez CT, McLendon L, Wisniewski KE, Jenkins EC, Brown WT. Molecular diagnosis of and carrier screening for the neuronal ceroid lipofuscinoses (NCLs). Genet Testing 2000; $4: 243-248$.

25. Zhong N. Molecular genetic testing for neuronal ceroid lipofuscinoses. In: Wisniewski KE, Zhong N, editors. Batten Disease: diagnosis, treatment, research. San Diego, CA: Academic Press, 2000, 217-224.

26. Hartikainen IM, Ju W, Wisniewski KE, Moroziewicz DN, McLendon L, Zhong D, Brown WT, Zhong N. Late infantile neuronal ceroid lipofuscinosis is due to splicing mutations in the CLN2 gene. Mol Genet Metab 1999;67:162-168.

27. Sleat DE, Gin RM, Sohar I, Wisniewski K, Sklower-Brooks S, Pullarkat RK, Palmer DN, Lerner TI, Boustany RM, Uldall P, Siakotos AN, Donnelly RJ, Lobel P. Mutational analysis of the defective protease in classic late-infantile neuronal ceroid lipofuscinosis, a neurodegenerative lysosomal storage disorder. Am I Hum Genet 1999; 64:1511-1523.

28. Junaid MA, Sklower-Brooks S, Wisniewski KE, Pullarkat RK. A novel assay for lysosomal pepstatin-insensitive proteinase and its application for the diagnosis of late-infantile neuronal ceroid lipofuscinosis. Clin Chim Acta 1999;281:169-176.

29. Savukoski M, Klockars T, Holmberg V, Santavuori P, Lander ES, Peltonen L. CLN5, a novel gene encoding a putative transmembrane protein mutated in Finnish variant late infantile neuronal ceroid lipofuscinosis. Nat Genet 1998;19:286-288. 


\section{Zhong et al.}

30. Sharp JD, Wheeler RB, Lake BD, Fox M, Gardiner RM, Williams RE. Genetic and physical mapping of the CLN6 gene on chromosome 15q21-23. Mol Genet Metab 1999;66:329-331.

31. Wheeler RB, Sharp JD, Mitchell WA, Bate SL, Williams RE, Lake BD, Gardiner RM A new locus for variant late infantile neuronal ceroid lipofuscinosis-CLN7. Mol Genet Metab 1999;66:337-338.

32. Ranta S, Zhang Y, Ross B, Lonka L, Takkunen E, Messer A, Sharp J, Wheeler R Kusumi K, Mole S, Liu W, Soares MB, Bonaldo MF, Hirvasniemi A, de la Chapelle A, Gilliam TC, Lehesjoki AE. The neuronal ceroid lipofuscinoses in human EPMR and mnd mutant mice are associated with mutations in CLN8. Nat Genet 1999;23:223226.
33. Sharp JD, Wheeler RB, Lake BD, Savukowski M, Jarvela IE, Peltonen L, Gardiner RM, Williams RE. Loci for classical and a variant late infantile neuronal ceroid lipofuscinoses map to chromosome 11p15 and 15q21-23. Hum Mol Genet 1997;6:591-596.

34. Boustany R-MN. Neurodystrophies and lipidosis. In: Moser H, editor. Handbook of clinical neurology, vol 2. Amsterdam: Elsevier, 1997:1-30.

35. Porter BE, Jacobs D, Chyung ASC, Zhong N, Solomon D, Liu GT, Tennekoon GI Ocular motor apraxia as a presenting symptom of a novel mutation in the lateinfantile neuronal ceroid lipofuscinosis (LINCL). Presented at the 29th Annual Meeting of Child Neurology Society. St. Louis, Missouri, October 25-28, 2000.

36. Zhong N, Moroziewicz DN, Ju W, Jurkiewciz A, Wisniewski KE, Brown WT. CLN gene-encoded proteins do not interact among each other. Neurogenetics 2000;3:4I-44. 